\title{
Ammonia removal using activated carbons: Effect of the surface chemistry in dry and moist conditions
}

\begin{tabular}{|r|l|}
\hline Journal: & Environmental Science \& Technology \\
\hline Manuscript ID: & es-2011-03093v.R1 \\
\hline Manuscript Type: & Article \\
\hline Author: & $\mathrm{n} / \mathrm{a}$ \\
\hline & $\begin{array}{l}\text { Complete List of Authors: } \\
\text { Sancheres-García, Laura; Facultad de Ciencias, Universidad de Alicante } \\
\text { Silvestre-Albero, Joaquin; University of Alicante, Inorganic Chemistry } \\
\text { Department } \\
\text { Rodriguez-Reinoso, Francisco; Facultad de Ciencias, Universidad de } \\
\text { Alicante }\end{array}$ \\
\hline
\end{tabular}

\section{SCHOLARONE Manuscripts}




\title{
Ammonia removal using activated carbons: Effect of the surface chemistry in dry and moist conditions
}

\author{
Maraisa Gonçalves, Laura Sánchez-García, Erika Oliveira-Jardim, Joaquín Silvestre-Albero*, \\ Francisco Rodríguez-Reinoso
}

\begin{abstract}
Laboratorio de Materiales Avanzados, Departamento de Química Inorgánica-Instituto Universitario de Materiales, Universidad de Alicante, Ap. 99, E-03080 Alicante, Spain
\end{abstract}

\footnotetext{
*Corresponding author: Joaquín Silvestre-Albero (joaquin.silvestre@ua.es)
}

Tel./Fax.: +34965909350/3454 


\begin{abstract}
The effect of surface chemistry (nature and amount of oxygen groups) in the removal of ammonia was studied using a modified resin-based activated carbon. $\mathrm{NH}_{3}$ breakthrough column experiments show that the modification of the original activated carbon with nitric acid, i.e. the incorporation of oxygen surface groups, highly improves the adsorption behaviour at room temperature. Apparently, there is a linear relationship between the total adsorption capacity and the amount of the more acidic and less stable oxygen surface groups. Similar experiments using moist air clearly show that the effect of humidity highly depends on the surface chemistry of the carbon used. Moisture highly improves the adsorption behaviour for samples with a low concentration of oxygen functionalities, probably due to the preferential adsorption of ammonia via dissolution into water. On the contrary, moisture exhibits a small effect on samples with a rich surface chemistry due to the preferential adsorption pathway via Brønsted and Lewis acid centers from the carbon surface. FTIR analyses of the exhausted oxidized samples confirm both the formation of $\mathrm{NH}_{4}{ }^{+}$species interacting with the Brønsted acid sites, together with the presence of $\mathrm{NH}_{3}$ species coordinated, through the lone pair electron, to Lewis acid sites on the graphene layers.
\end{abstract}

KEYWORDS: Activated carbon, ammonia, surface chemistry. 


\section{Introduction}

New air quality emission standards are forcing the different governments to adopt new regulations in order to control pollutant emissions. Among them, ammonia is considered an important health hazard because it is poisonous if inhaled in great quantities (breathing levels above 50-100 ppm) while it can cause eyes, throat and nose irritation in lesser concentrations. ${ }^{1}$

Actual ammonia emissions are generated mainly from the fertilizer manufacture industry, coke manufacture, fossil fuel combustion, livestock and poultry management, and refrigeration methods. Among all these sources, livestock waste management and fertilizers production account for about $90 \%$ of total ammonia emissions. ${ }^{1,2}$ Many techniques have been proposed in the literature for the removal of $\mathrm{NH}_{3}$ in industrial effluents. These include absorption by solution, reaction with other gases, ion exchange using polymeric resins, separation using membranes, thermal treatment, catalytic decomposition and adsorption by porous solids. ${ }^{3-5}$ Some of these techniques (e.g. thermal combustion) are economically convenient for high concentration pollutants, whereas they become economically unviable for diluted waste streams. For these special cases adsorption on porous solids (e.g. activated carbons, zeolites, and so on) can be an excellent approach. ${ }^{6-12}$ Among the different porous solids described in the literature, activated carbons exhibit certain advantages such as a high "apparent" surface area, a highly developed porous structure and, most importantly, the possibility to tailor the porous structure and surface chemistry in order to adapt it for a special application. ${ }^{13}$ These modifications using pre- and post-synthesis treatments are very convenient when trying to adsorb polar gases (e.g. $\mathrm{NH}_{3}$ ), because activated carbons usually exhibit non-polar surfaces and surface modifications are mandatory.

Previous studies described in the literature indicated that the surface chemistry is probably the most critical parameter defining the total adsorption capacity of activated carbons for a basic molecule such as ammonia. ${ }^{6,7,9}$ Kim et al. showed that not only the amount but also the nature of the oxygen surface groups present on the carbon surface, i.e. acidic groups, are responsible for the total amount adsorbed. ${ }^{7}$ Furthermore, Huang et al. reported a linear correlation between $\mathrm{NH}_{3}$ breakthrough capacity and the total 
amount of acidic groups. ${ }^{6}$ A similar beneficial effect for the ammonia removal has been described in the literature for metal-modified (Fe, Co, Cr, Mo and W) activated carbons. ${ }^{8,10,14}$

Different reaction mechanisms have been proposed for the adsorption process involving mainly Brønsted acid sites, but the true pathway is still under debate. Unfortunately, many of these studies described in the literature deal with a wide variety of carbon samples, i.e. different textural and chemical properties, together with additional effects due to the presence of metal species, which makes difficult a clear correlation between the inherent parameters of the carbon support and the adsorption mechanism. ${ }^{8,10,14}$ Furthermore, only few studies have paid attention to the role of moisture usually present in industrial streams.

The objective of this paper is to evaluate the real role of the oxygen surface groups present on the surface of activated carbons in the removal of ammonia, either in the presence or absence of moisture. For this purpose, an activated carbon prepared from a resin precursor was modified by an oxidation treatment with $\mathrm{HNO}_{3}$, followed by a subsequent thermal treatment at different temperatures in order to selectively remove some of the oxygen surface groups. Compared to previous analysis described in the literature, the sole modification of the surface chemistry in a controlled way will allow to analyze the effect of the surface chemistry in the ammonia adsorption process, under dry or moist conditions, avoiding the influence of additional parameters (e.g. porous structure or inorganic matter).

\section{Experimental Section}

\subsection{Materials}

A spherical activated carbon (MA2, supplied by MAST Carbon International, UK) with particle size around $0.32 \mathrm{~mm}$ was prepared by carbonization and subsequent activation using $\mathrm{CO}_{2}$ (43\% burn-off) of a porous resin obtained by cross-linking of phenol-formaldehyde Novolac precursor with hexamethylenetetramine and using ethylene glycol as solvent-pore former. For the oxidation treatment, $25 \mathrm{~g}$ of carbon were treated with $250 \mathrm{ml} \mathrm{HNO}_{3}$ solution $(6 \mathrm{M})$ at $90^{\circ} \mathrm{C}$ for $1 \mathrm{~h}$. The oxidized sample (MA2ox) was washed until neutral $\mathrm{pH}$ and dried overnight at $85^{\circ} \mathrm{C}$. In order to selectively remove the 
surface functional groups, sample MA2ox was submitted to a subsequent thermal treatment at 300, 500 and $700^{\circ} \mathrm{C}$ under a helium flow $(50 \mathrm{ml} / \mathrm{min})$ for $1 \mathrm{~h}$. Samples were labelled MA2ox300, MA2ox500 and MA2ox700, respectively.

\subsection{Characterization}

$\mathrm{N}_{2}$ adsorption/desorption isotherms were performed at $-195^{\circ} \mathrm{C}$ in a home-made high precision volumetric equipment. Before any experiment samples were degassed under vacuum $\left(10^{-3} \mathrm{~Pa}\right)$ at $150^{\circ} \mathrm{C}$ for $4 \mathrm{~h}$. "Apparent" surface area was calculated from the nitrogen adsorption data after application of the BET equation while the micropore volume $\left(\mathrm{V}_{0}\right)$ was obtained after application of the DubininRadushkevich (DR) equation. $\mathrm{CO}_{2}$ adsorption isotherms were performed in the same apparatus at $0^{\circ} \mathrm{C}$ following the same protocol. Application of the DR equation to the $\mathrm{CO}_{2}$ adsorption data was used to calculate the volume of narrow micropores $\left(V_{n}\right)$, i.e. those below $0.7 \mathrm{~nm}^{15}$

Temperature programmed decomposition experiments (TPD) were performed in order to evaluate the amount and nature of oxygen surface functionalities for the different samples. $100 \mathrm{mg}$ of carbon were placed in a quartz reactor under a helium flow $(50 \mathrm{ml} / \mathrm{min})$ and submitted a heat treatment up to $1000^{\circ} \mathrm{C}$ $\left(10^{\circ} \mathrm{C} / \mathrm{min}\right)$. The different gas species evolved as a result of surface groups decomposition (mainly $\mathrm{CO}$ and $\mathrm{CO}_{2}$ ) were analyzed using an on-line mass spectrometer (Omnistar TM, Balzers). Quantitative analyses were performed after calibration using $\mathrm{CaC}_{2} \mathrm{O}_{4} \cdot \mathrm{H}_{2} \mathrm{O}$ as a reference material.

FTIR spectra of the exhausted samples were obtained in the range $4000-700 \mathrm{~cm}^{-1}$, by adding 100 scans at a resolution of $5 \mathrm{~cm}^{-1}$, using a Mattson Infinity Gold FTIR spectrometer in the diffuse reflectance method. Before any experiment all samples were diluted with $\mathrm{KBr}$.

\subsection{Breakthrough column experiments}

The adsorption capacity under dynamic conditions for ammonia removal at room temperature $\left(23^{\circ} \mathrm{C}\right)$ was measured using a $1 \mathrm{~cm}$ i.d. column containing $2 \mathrm{~cm}$ carbon bed height ( $\sim 0.6 \mathrm{~g}$ of sorbent). A total flow of $300 \mathrm{ml} / \mathrm{min}$ of air containing $1000 \mathrm{ppm}$ of ammonia was passed through the column containing 
the fresh sample (without any pre-treatment). The concentration of ammonia at the exit was followed using a Polytron 3000 (Drager) detector. Adsorption experiments were performed using either dry or moist air (70\% humidity). For moist conditions, humidity was introduced into the $\mathrm{NH}_{3} /$ air flow using a calibrated syringe. The breakthrough experiment was arbitrarily stopped when the ammonia concentration at the outlet reached a concentration of $100 \mathrm{ppm}$. The adsorption capacity was determined by integration of the area above the breakthrough curve and considering the inlet concentration, the total flow rate and the amount of sorbent used. To further clarify the role of humidity, additional experiments were performed using pre-humidified samples $(20 \%, 40 \%$ and $70 \%$ relative humidity). For this purpose, the carbon samples were first submitted to a thermal treatment at $100^{\circ} \mathrm{C}$ overnight in order to remove the residual humidity and, in a subsequent step, samples were exposed to a given $\mathrm{H}_{2} \mathrm{O} / \mathrm{H}_{2} \mathrm{SO}_{4}$ mixture for $24 \mathrm{~h}$ at room temperature under controlled humidity conditions. Breakthrough column experiments using the pre-humidified samples were always performed under dry conditions.

\section{Results and discussion}

\subsection{Sample characterization}

Figure 1 shows the nitrogen adsorption/desorption isotherms for the original and the modified activated carbon. As it can be observed, the resin-based activated carbon (MA2) exhibits a highly developed microporous structure, i.e. closed knee in the nitrogen adsorption isotherm at low relative pressure, together with capillary condensation processes at $\mathrm{p} / \mathrm{p}_{0} \sim 0.8$, corresponding to the filling of large mesopores. The oxidation treatment with $\mathrm{HNO}_{3}$ produces slight changes in the porous structure, the main effect being the partial blockage of the microporosity due to the creation of polar oxygen surface groups at the entrance of narrow pores; this slightly decreases the adsorption of a molecule such as nitrogen, due to steric effects at the pore mouth. ${ }^{16,17} \mathrm{~A}$ subsequent thermal treatment at high temperature $\left(700^{\circ} \mathrm{C}\right)$ under a helium flow produces the widening of the existing micro/mesopores due to the partial gasification of the carbon structure. ${ }^{13,18}$ The textural properties of the modified activated 
carbon obtained from the $\mathrm{N}_{2}$ and $\mathrm{CO}_{2}$ adsorption data, after application of the corresponding equations, are reported in Table 1.

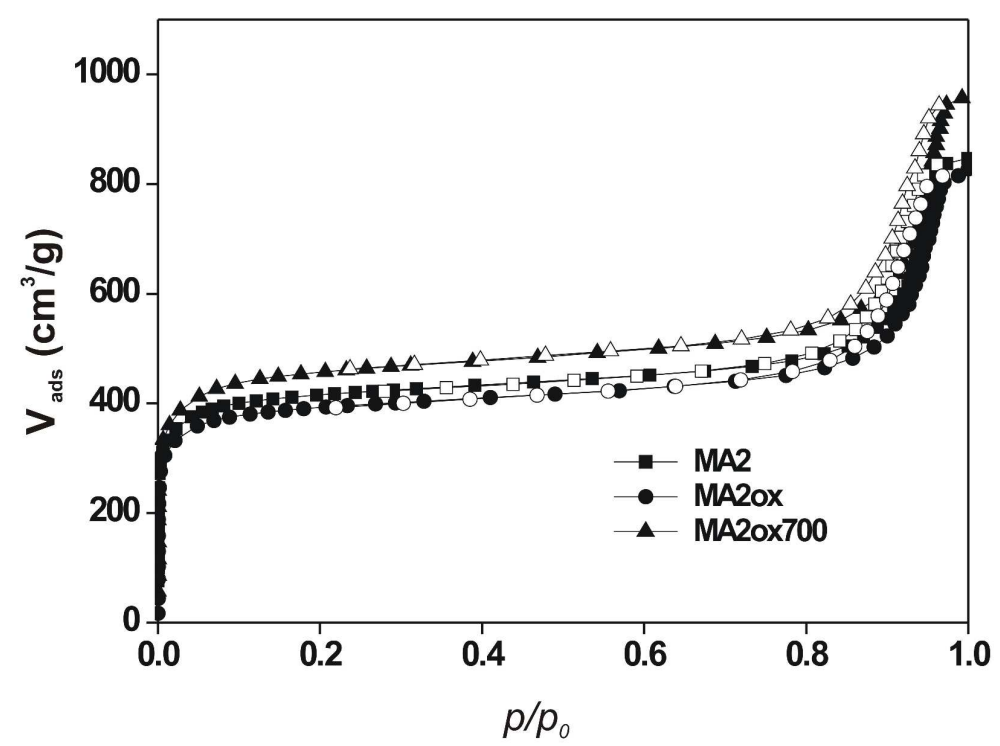

Figure 1. Nitrogen adsorption/desorption isotherms at $-195^{\circ} \mathrm{C}$ for the different activated carbons.

Table 1. Textural parameters for the different activated carbons obtained from the $\mathrm{N}_{2}$ and $\mathrm{CO}_{2}$ adsorption data at $-195^{\circ} \mathrm{C}$ and $0^{\circ} \mathrm{C}$, respectively.

\begin{tabular}{cccccc}
\hline Samples & $\begin{array}{c}\mathrm{S}_{\mathrm{BET}} \\
\left(\mathrm{m}^{2} / \mathrm{g}\right)\end{array}$ & $\begin{array}{c}\mathrm{V}_{0} \\
\left(\mathrm{~cm}^{3} / \mathrm{g}\right)\end{array}$ & $\begin{array}{c}\mathrm{V}_{\text {meso }} \\
\left(\mathrm{cm}^{3} / \mathrm{g}\right)\end{array}$ & $\begin{array}{c}\mathrm{V}_{\mathrm{t}(0.97)} \\
\left(\mathrm{cm}^{3} / \mathrm{g}\right)\end{array}$ & $\begin{array}{c}\mathrm{V}_{\mathrm{n}}{ }^{*} \\
\left(\mathrm{~cm}^{3} / \mathrm{g}\right)\end{array}$ \\
\hline MA2 & 1550 & 0.61 & 0.68 & 1.29 & 0.55 \\
MA2ox & 1470 & 0.61 & 0.63 & 1.24 & 0.53 \\
MA20x300 & 1570 & 0.63 & 0.67 & 1.30 & 0.54 \\
MA20x500 & 1580 & 0.64 & 0.67 & 1.31 & 0.54 \\
MA20x700 & 1700 & 0.69 & 0.74 & 1.43 & 0.61 \\
\hline "Obtained after application of the D-R equation to the $\mathrm{CO}_{2}$ adsorption data at $0^{\circ} \mathrm{C}$
\end{tabular}

The surface chemistry of the original and modified activated carbons was evaluated using temperature-programmed decomposition (TPD) experiments. The amount of $\mathrm{CO}_{2}$ and $\mathrm{CO}$ evolved in the temperature range from room temperature up to $1000^{\circ} \mathrm{C}$ was analyzed as described in the experimental 
section. Quantitative data for the different samples are compiled in Table 2. $\mathrm{CO}_{2}$ groups usually evolve at low temperatures and they correspond to the decomposition of the more acidic and less stable oxygen surface groups (mainly carboxylic and lactone groups), while $\mathrm{CO}$ evolution is due to the decomposition of the more stable and less acidic oxygen groups (mainly phenolic, carbonyl and quinone groups) and takes place at a higher temperature. ${ }^{19}$

Table 2. Total amount of $\mathrm{CO}_{2}$ and $\mathrm{CO}$ groups evolved in the TPD experiment.

\begin{tabular}{ccc}
\hline Carbons & $\begin{array}{c}\mathrm{CO} \\
(\mathrm{mmol} / \mathrm{g})\end{array}$ & $\begin{array}{c}\mathrm{CO}_{2} \\
(\mathrm{mmol} / \mathrm{g})\end{array}$ \\
\hline MA2 & 0.837 & 0.18 \\
MA2ox & 2.800 & 1.770 \\
MA2ox300 & 2.820 & 0.862 \\
MA2ox500 & 1.822 & 0.245 \\
MA2ox700 & 1.048 & 0.093 \\
\hline
\end{tabular}

The as-received activated carbon (MA2) shows a relatively poor surface chemistry, i.e. it has a small amount of oxygen surface groups, with a larger content of the less acidic groups compared to those evolved as $\mathrm{CO}_{2}\left(\mathrm{CO} / \mathrm{CO}_{2}\right.$ ratio of 4.6). As expected, the oxidation treatment with nitric acid produces a large increase in the amount of both types of oxygen surface groups, this enhancement being larger for the $\mathrm{CO}_{2}$ groups $\left(\mathrm{CO} / \mathrm{CO}_{2}\right.$ ratio of 1.6$)$, in accordance with previous observations. ${ }^{17} \mathrm{~A}$ subsequent thermal treatment at low temperature $\left(300^{\circ} \mathrm{C}\right)$ produces an important decrease in the amount of the less stable and more acidic oxygen groups, i.e. those evolved as $\mathrm{CO}_{2}$, while the more stable groups remain mainly unaffected. Temperatures higher than $300^{\circ} \mathrm{C}$ are required to selectively remove not only the more acidic but also the majority of the more stable oxygen surface groups, i.e. those evolved as $\mathrm{CO}$ in the TPD experiment. 


\subsection{Breakthrough column experiments}

Breakthrough column experiments were performed in a glass tubular reactor using a total flow of 300 $\mathrm{ml} / \mathrm{min}$ of either dry or moist air (70\% humidity) containing $1000 \mathrm{ppm} \mathrm{NH}_{3}$. Figure 2 shows the corresponding curves obtained using (a) dry air and (b) moist conditions. The total adsorption capacity (mg/g) for the different samples is reported in Table 3.
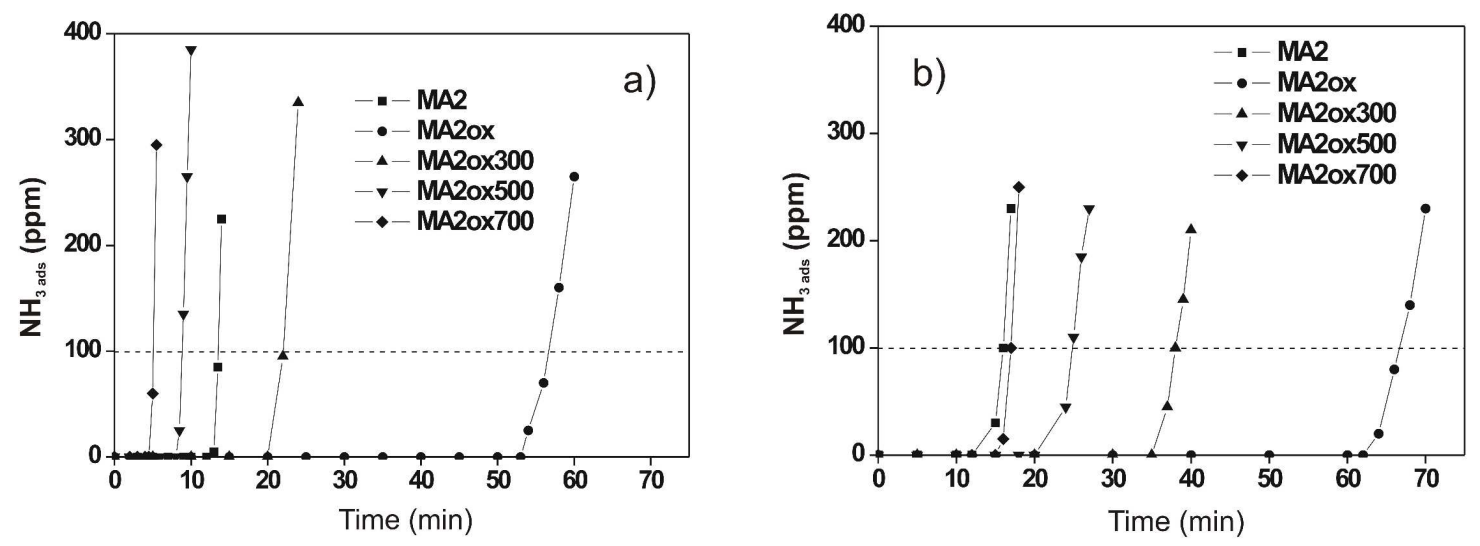

Figure 2. Breakthrough curves of ammonia adsorbed on the different activated carbons (1000 ppm, $300 \mathrm{ml} / \mathrm{min}, 23^{\circ} \mathrm{C}$ ) using (a) dry and (b) moist (70\% relative humidity) air.

Dynamic adsorption experiments show important differences among the different samples either in the presence or absence of moisture. In general all samples exhibit a sharp breakthrough saturation profile that proves the absence of kinetic restrictions in the adsorption process. This observation must be attributed to fast adsorption kinetics of $\mathrm{NH}_{3}$ through the mesoporous network on these carbon materials, since the mesopores constitute the channels to access the inner microporosity. Under dry conditions (see Figure 2a) the total adsorption capacity largely improves (close to five fold increase) after an oxidation treatment of the original carbon with $\mathrm{HNO}_{3}$. The total adsorption capacity for the oxidized sample (MA2ox) achieves a value as high as $17.5 \mathrm{mg} / \mathrm{g}$, in close agreement with previous measurements reported in the literature for oxidized activated carbons. ${ }^{6,8,20}$ A subsequent thermal treatment at low and high temperature produces a large decrease in the uptake down to a residual value even lower than the one of the original sample. Interestingly, the decline in the adsorption capacity is very drastic after the 
thermal treatment at low temperature (sample MA2ox300), whereas a small decrease is observed thereafter (up to $700^{\circ} \mathrm{C}$ ). This observation anticipates the crucial role of the oxygen surface groups in the gas phase removal of ammonia and, more specifically, the important effect of the more acidic and less stable (those decomposing at low temperature) oxygen surface groups (mainly carboxylic groups) in the adsorption process under dry conditions. ${ }^{6,7}$ Incorporation of $70 \%$ relative humidity in the inlet stream has a different effect depending on the surface chemistry (Figure 2b). The oxidized sample (MA2ox) exhibits a moderate improvement in the adsorption behaviour after moisture incorporation, this clearly suggesting the predominant role of the oxygen surface groups in the adsorption process, i.e. water must exhibit a marginal effect in the adsorption mechanism. Concerning the thermally treated samples, Figure $2 \mathrm{~b}$ shows that the improvement in the adsorption capacity due to moisture incorporation is larger compared to the oxidized sample and it remains mainly constant for all samples, independently of the temperature of the thermal treatment applied (see Table 3). Apparently on these thermally treated samples, i.e. these samples were the more acidic and less stable oxygen surface groups have been removed, moisture exhibits a crucial role in the adsorption mechanism, independently of the amount and nature of the remaining surface functionalities. On the contrary, on carbons with a rich surface chemistry the preferential interaction of ammonia with the carbon surface, assisted by the oxygen surface groups and, more specifically, by the more acidic groups, could be the key factor defining the total adsorption capacity, independently of the presence or absence of moisture. The large adsorption capacity achieved with the oxidized sample (MAox) under dry conditions, together with the slight improvement observed after moisture incorporation is somehow in contradiction with previous studies described in the literature, which proposed a crucial role of water in the formation of $\mathrm{NH}_{4}{ }^{+}$ions, necessary for the adsorption process via interaction with the Brønsted acid groups of the carbon surface. ${ }^{8}$ 
Table 3. Total adsorption capacity for ammonia under dry and moist (70\% relative humidity) air conditions.

\begin{tabular}{cccc}
\hline Carbons & $\begin{array}{c}\mathrm{NH}_{3} \text { dried } \\
(\mathrm{mg} / \mathrm{g})\end{array}$ & $\begin{array}{c}\mathrm{NH}_{3} 70 \% \mathrm{RH} \\
(\mathrm{mg} / \mathrm{g})\end{array}$ & $\begin{array}{c}\Delta \mathrm{m}(\mathrm{mg} / \mathrm{g}) \\
(0-70 \% \mathrm{RH})\end{array}$ \\
\hline MA2 & 4.7 & 5.3 & 0.6 \\
MA20x & 17.5 & 20.1 & 2.6 \\
MA20x300 & 7.6 & 12.3 & 4.7 \\
MA20x500 & 3.4 & 7.6 & 4.2 \\
MA20x700 & 1.9 & 6.1 & 4.2 \\
\hline
\end{tabular}

To further clarify the real role of the oxygen surface groups in the adsorption process Figure 3a compares the total adsorption capacity, either under dry or moist conditions, and the total amount of acidic oxygen surface groups, i.e. those decomposing as $\mathrm{CO}_{2}$ in a typical TPD run. As described above, the total adsorption capacity is larger in the presence of moist air for all samples, independently of the surface chemistry. However, contrary to previous analysis described in the literature, the presence of moisture does not seem to be so critical for the ammonia adsorption process when oxygen surface groups, mainly acidic, are present (e.g. sample MAox); in other words, the adsorption capacity of the oxidized sample under dry conditions is rather high and the improvement after moisture incorporation is rather small. ${ }^{8}$ Interestingly, there is a linear correlation between the amount of ammonia adsorbed and the total amount of acidic groups on the carbon surface, either in the presence or absence of humidity. Furthermore, a closer look to Figure 3a shows that the beneficial effect of moisture slightly decreases when the amount of oxygen surface groups increases, thus suggesting a different adsorption mechanism in the presence or absence of surface functionalities (mainly acidic oxygen surface groups). While moisture highly improves the adsorption behaviour of the carbon materials with a poor surface chemistry (69\% improvement on sample MA2ox700), moisture seems to exhibit a minor effect (13\% improvement on sample MA2ox) in the presence of oxygen functionalities (mainly acidic groups), probably due to the preferential adsorption mechanism involving these oxygen surface groups. 
Incorporation of oxygen functionalities to the carbon surface can modify the adsorption behaviour through two different mechanisms: i) the creation of active sites, preferentially acidic oxygen groups, at the periphery of the basal planes, which will promote $\mathrm{NH}_{3}$ adsorption via a Brønsted acid-base process through ammonium ion formation $\left(\mathrm{NH}_{4}{ }^{+}\right)$or ii) the creation of Lewis acid centers on the graphene layers due to the withdraw of electron density by the more electronegative oxygen surface groups on the periphery, thus promoting the interaction of ammonia (Lewis base) via the lone pair electron. Taking into account that the incorporation of the oxygen functionalities to the carbon surface promotes both processes at the same time, the presence of a linear correlation as observed in Figure 3a does not provide enough information to exclude one mechanism against the other.

Previous studies described in the literature using a large variety of activated carbons have shown that when the adsorption process is governed by a pure Brønsted acid-base process, the adsorption behaviour increases exponentially below a certain $\mathrm{pH}(\sim 4.5)$, which corresponds to the $\mathrm{pK}_{\mathrm{a}}$ for dissociation of carboxylic acid groups on the carbon surface. ${ }^{8}$ In this sense, Figure $3 b$ shows the relationship between the total amount adsorbed and the surface $\mathrm{pH}$ of the different samples used. At this point it is important to highlight that at the $\mathrm{pH}$ of all activated carbons used in this manuscript $(\mathrm{pH}<6.5), \mathrm{NH}_{3}$ will be protonated to $\mathrm{NH}_{4}{ }^{+}$in a proportion close to $100 \%$ ( $\mathrm{pk}_{\mathrm{a}}$ for ammonia is 9.3 ). As it can be observed, the absence of an exponential increase in the amount adsorbed below a certain $\mathrm{pH}$ value, either in the presence or absence of moisture, clearly suggests that the $\mathrm{NH}_{3}$ adsorption mechanism on activated carbons is not exclusively governed by the Brønsted acid sites on the surface, as proposed in the literature. ${ }^{8}$ Most probably, both adsorption mechanisms, i.e. Lewis and Brønsted mechanisms, take place on activated carbons even in the presence or absence of moisture. 

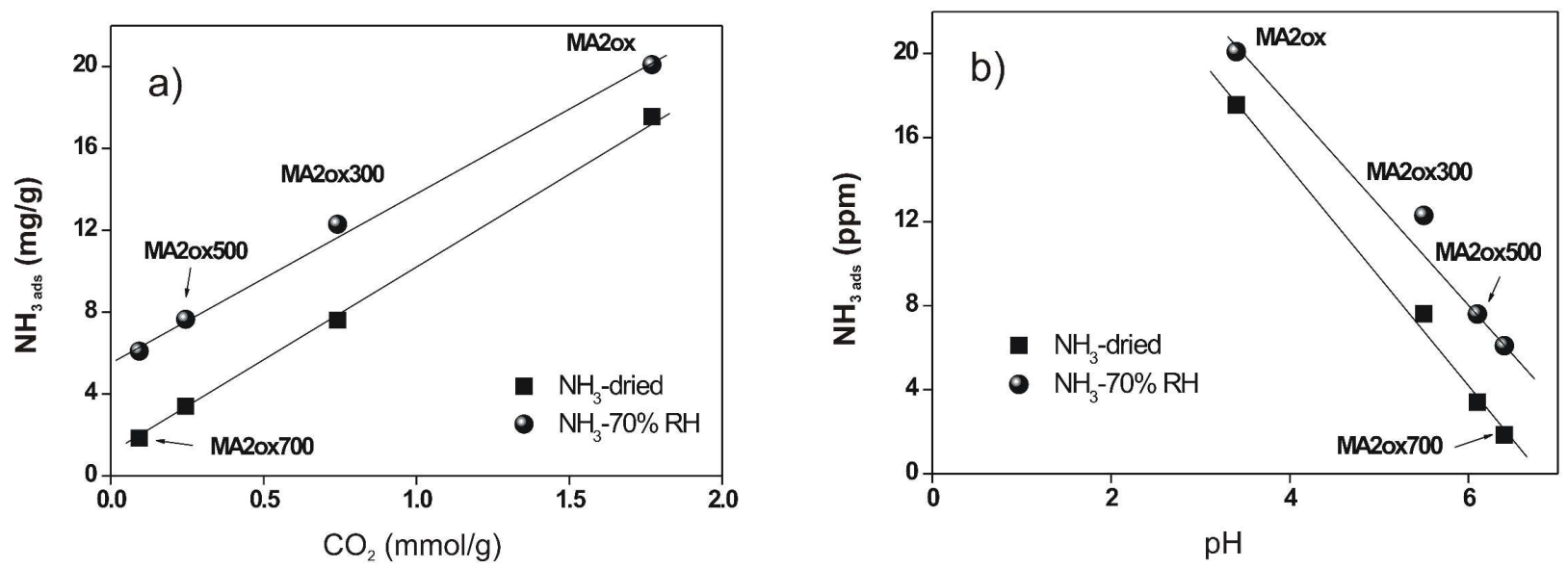

Figure 3. Dependence of the total breakthrough column capacity (a) as a function of the amount of acidic oxygen surface groups and (b) as a function of surface $\mathrm{pH}$, in the presence or absence of moisture.

To further clarify the role of humidity, a new series of experiments were performed using prehumidified samples. In a first step, carbon materials were heat treated at $100^{\circ} \mathrm{C}$ overnight in order to remove the residual humidity; in a second step, activated carbons were submitted to a pre-humidification treatment at different percentages $\left(20 \%, 40 \%\right.$ and $70 \%$ relative humidity) using a $\mathrm{H}_{2} \mathrm{O} / \mathrm{H}_{2} \mathrm{SO}_{4}$ mixture and, in a final step, carbon samples were analyzed in ammonia breakthrough column experiments using dry air. Figure 4 reports the amount of $\mathrm{NH}_{3}$ adsorbed for samples MA2ox and MA2ox700, i.e. samples with a completely different surface chemistry, under different pre-humidification conditions and as a function of the weight gain after the pre-humidification step, i.e. the amount of humidity retained by the sample. As expected, the oxidized sample (MA2ox) is more hydrophilic and, consequently, it is able to absorb a larger amount of water under the pre-humidification conditions used. Figure 4 shows that for the sample with a poor surface chemistry (sample MA2ox700) there is a linear relationship between the amount of water adsorbed by the carbon sample and the subsequent adsorption capacity for a basic molecule such as ammonia. This linear relationship confirms that moisture plays a crucial role in the adsorption behaviour on this sample. Most probably in samples with a poor surface chemistry (absence of acidic groups), adsorption of ammonia takes place via dissolution into water, in such a way that the 
amount of ammonia dissolved increases with the amount of solvent. The total adsorption capacity ranges from $1.1 \mathrm{mg} / \mathrm{g}$ for the dried sample to $5.3 \mathrm{mg} / \mathrm{g}$ for the sample pre-humidified with $70 \% \mathrm{RH}$, this meaning a 4.8 fold increase. On the contrary, the oxidized sample exhibits a completely different behaviour. As described above, the adsorption capacity of the untreated sample, i.e. in the absence of humidity, is as large as $15.6 \mathrm{mg} / \mathrm{g}$, thus confirming the crucial role of the oxygen surface groups in the adsorption of a basic molecule such as ammonia. At this point it must be highlight that this value is slightly smaller than the one reported in Table $3(17.5 \mathrm{mg} / \mathrm{g}$ vs $15.6 \mathrm{mg} / \mathrm{g})$ for the same sample, thus confirming the beneficial effect of the inherent humidity on the fresh sample. Pre-humidification with $20 \% \mathrm{RH}$ produces a slight increase in the adsorption capacity up to a value of $17.5 \mathrm{mg} / \mathrm{g}$, remaining constant thereafter (up to $70 \% \mathrm{RH}$ ). Taking into account that the amount of water adsorbed highly increases with the pre-humidification step (from 0 to $70 \% \mathrm{RH}$ ) on sample MA2ox, the absence of significant changes in the adsorption capacity for this sample above $20 \% \mathrm{RH}$ clearly suggests that the adsorption mechanism in carbons with a rich surface chemistry follows a different pathway. Under these conditions moisture exhibits a scarce effect, the main contribution to the adsorption process coming from the oxygen groups, mainly acidic groups, present on the carbon surface as suggested in Figure 3 a. As described above, the adsorption mechanism on oxidized samples can proceed following two approaches: i) by interaction of $\mathrm{NH}_{3}$ through the lone pair electron with the Lewis acidic sites on the graphene planes; or ii) by interaction of $\mathrm{NH}_{3}$ with acidic oxygen surface groups via $\mathrm{NH}_{4}{ }^{+}$ions formation. Apparently, both adsorption mechanisms take place in the oxidized samples either in the presence and absence of moisture, although with a certain prevalence for the Lewis acid-base mechanism, according to Figure $3 b$. 


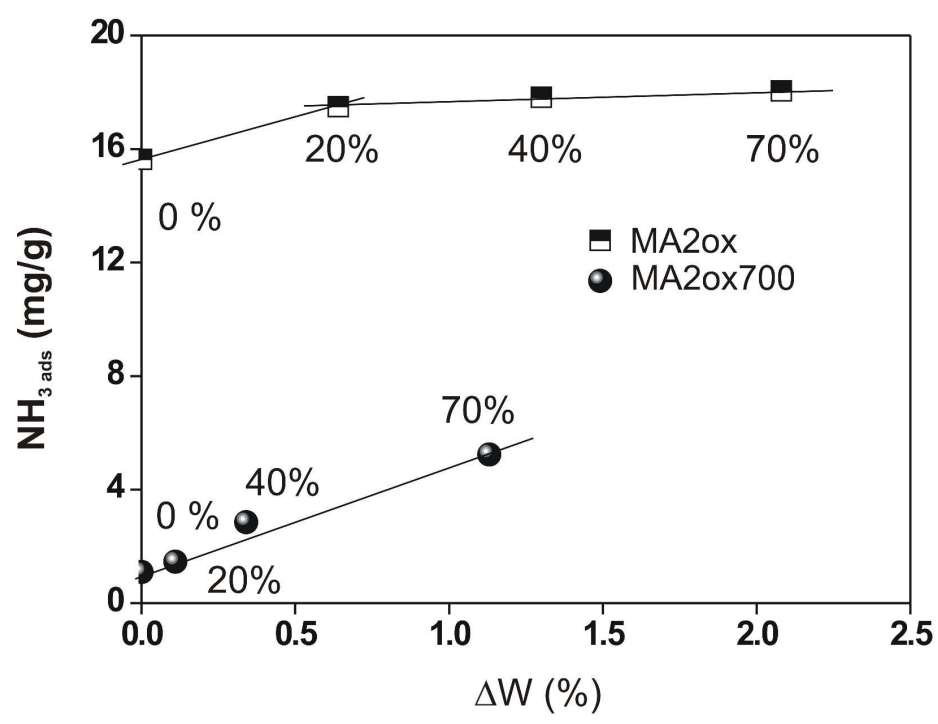

Figure 4. Dependence of ammonia adsorption capacity on the amount of water adsorbed under different humidity conditions (RH 0-70 \%) for samples MA2ox and MA2ox700.

In a final step, the nature of the active species after the adsorption process has been analyzed on the exhausted samples using FTIR. As it can be observed in Figure 5, the heat-treated sample MA2ox700 exhibits a poor FTIR profile, i.e. the amount of surface species after the adsorption process is very small, in accordance with the above breakthrough column tests. FTIR spectra of the oxidized sample, MA2ox, after the adsorption process show intense bands, mainly when the experiment is performed in the presence of moisture. Mutually overlapping bands of $\mathrm{OH}$ and $\mathrm{NH}$ stretching vibration appear as a broad contribution at $3271 \mathrm{~cm}^{-1}$ as a result of $\mathrm{NH}_{3}$ adsorption. Furthermore, bands at $1637 \mathrm{~cm}^{-1}$ and $1082 \mathrm{~cm}^{-1}$, $\delta_{\text {as }} \mathrm{NH}_{3}$ and $\delta_{\text {sym }} \mathrm{NH}_{3}$, respectively, denote the presence of $\mathrm{NH}_{3}$ coordinated to Lewis acid sites on the carbon surface (graphene layers). ${ }^{11}$ Formation of surface ammonium salts of carboxylic acid appears as a band at $1439 \mathrm{~cm}^{-1}\left(\delta_{\mathrm{sym}} \mathrm{NH}_{4}^{+}\right.$ion $)$, as well as bands at $1521 \mathrm{~cm}^{-1}\left(v_{\mathrm{as}} \mathrm{COO}^{-}\right)$and $1393-1236 \mathrm{~cm}^{-1}\left(v_{\mathrm{sym}}\right.$ $\mathrm{COO}^{-}$). Consequently, FTIR analysis of the exhausted samples confirms the presence of two different adsorption mechanisms for ammonia adsorption on oxidized activated carbons: adsorption on Brønsted acid sites (carboxylic acid sites), via ammonium ion $\left(\mathrm{NH}_{4}^{+}\right)$formation, and ii) adsorption on Lewis acid 
sites on the graphene layers, via the lone pair electron of the ammonia molecule. Interestingly, both mechanisms dominate in oxidized carbon samples either in the presence or absence of moisture.

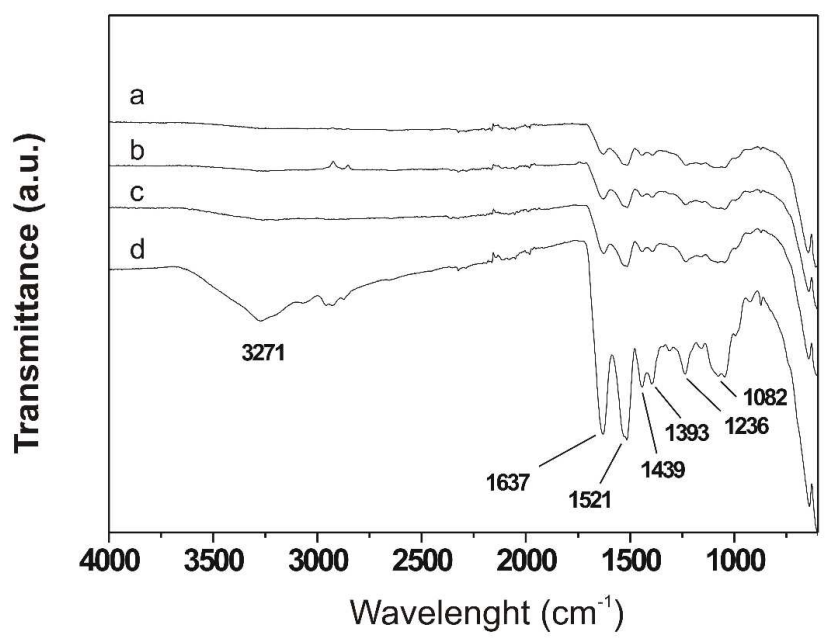

Figure 5. FTIR spectra of activated carbons (a) MA2ox700, (b)MA2ox700-70\% RH, (c) MA2ox, (d) MA2ox-70\% RH, after $\mathrm{NH}_{3}$ breakthrough column experiments.

\section{ACKNOWLEDGMENT}

Authors acknowledge financial support from MEC (projects MAT2007-61734 FEDER) and Generalitat Valenciana (PROMETEO/2009/002). European Commission is also acknowledge (project FRESP CA, contract 218138). J.S.A. acknowledges support from MEC, GV and UA (RyC2137/06).

\section{REFERENCES}

(1) Phillips, J. Control and pollution prevention options for ammonia emissions, EPA-456/R-95002, 1995.

(2) Calvert, S.; Englund, H. M. Handbook of Air Pollution Technology; Willey: New York, 1984. (3) Pez, G. P.; Laciak, D. V. Ammonia Separation using semipermeable membranes, US patent $\mathrm{n}$ 4,762,535, 1988.

(4) Blonigen, S. J.; Fassbender, A. G.; Litt, R. D.; Monzyk, B. F.; Neff, R. Method for ammonia removal from waste streams, US patent n 6,558,643, 2003.

(5) Blonigen, S. J.; Fassbender, A. G.; Litt, R. D.; Monzyk, B. F.; Neff, R. Apparatus and method for ammonia removal from waste streams, US patent n 6,838,069, 2005.

(6) Huang, C.-C.; Li, H.-S.; Chen, C.-H. Effect of surface acidic oxides of activated carbon on adsorption of ammonia. J. Hazard. Mater. 2008, 159, 523-527.

(7) Kim, B.-J.; Park, S.-J. Effect of carbonyl group formation on ammonia adsorption of porous carbon surfaces. J. Colloid Interface Sci. 2007, 311, 311-314. 
(8) Le Leuch, L. M.; Bandosz, T. J. The role of water and surface acidity on the reactive adsorption of ammonia on modified activated carbons. Carbon 2007, 45, 568-578.

(9) Park, S.-J.; Jin, S.-Y. Effect of ozone treatment on ammonia removal of activated carbons. $J$. Colloid Interface Sci. 2005, 286, 417-419.

(10) Petit, C.; Bandosz, T. J. Role of surface heterogeneity in the removal of ammonia from air on micro/mesoporous activated carbons modified with molybdenum and tungsten oxides. Microp. Mesop. Mater. 2009, 118, 61-67.

(11) Zawadzki, J.; Wisniewski, M. In situ characterization of interaction of ammonia with carbon surface in oxygen atmosphere. Carbon 2003, 41, 2257-2267.

(12) Bernal, M. P.; Lopez-Real, J. M. Natural zeolites and sepiolite as ammonium and ammonia adsorbent materials. Bioresource Technology 1993, 43, 27-33.

(13) Marsh, H.; Rodriguez-Reinoso, F. Activated Carbon; Elsevier: London, 2006.

(14) Bandosz, T. J.; Petit, C. On the reactive adsorption of ammonia on activated carbons modified by impregnation with inorganic compounds. J. Colloid Interface Sci. 2009, 338, 329-345.

(15) Garrido, J.; Linares-Solano, A.; Martín-Martínez, J. M.; Molina-Sabio, M.; Rodríguez-Reinoso, F.; Torregrosa, R. Use of nitrogen vs. carbon dioxide in the characterization of activated carbons.

Langmuir 1987, 3, 76-81.

(16) Rodríguez-Reinoso, F.; Molina-Sabio, M.; Muñecas-Vidal, M. A. Effect of microporosity and oxygen surface groups of activated carbon in the adsorption of molecules of different polarity. J. Phys. Chem. 1992, 96, 2707-2713.

(17) Silvestre-Albero, A.; Silvestre-Albero, J.; Sepúlveda-Escribano, A.; Rodríguez-Reinoso, F. Ethanol removal using activated carbon: Effect of porous structure and surface chemistry. Microp. Mesop. Mater. 2009, 120, 62-68.

(18) Molina-Sabio, M.; Muñecas, M. A.; Rodríguez-Reinoso, F. Modification in porous texture and oxygen surface groups of activated carbons by oxidation. Stud. Surf. Sci. \& Catal. 1991, 62, 329-339.

(19) Figueiredo, J. L.; Pereira, M. F. R.; Freitas, M. M. A.; Orfao, J. J. M. Modification of the surface chemistry of activated carbons. Carbon 1999, 37, 1379-1389.

(20) Rodrigues, C. C.; Moraes Jr., D. d.; da Nóbrega, S. W.; Barboza, M. G. Ammonia adsorption in a fixed bed activated carbon. Bioresource Technology 2007, 98, 886-891. 


\section{SYNOPSIS TOC}

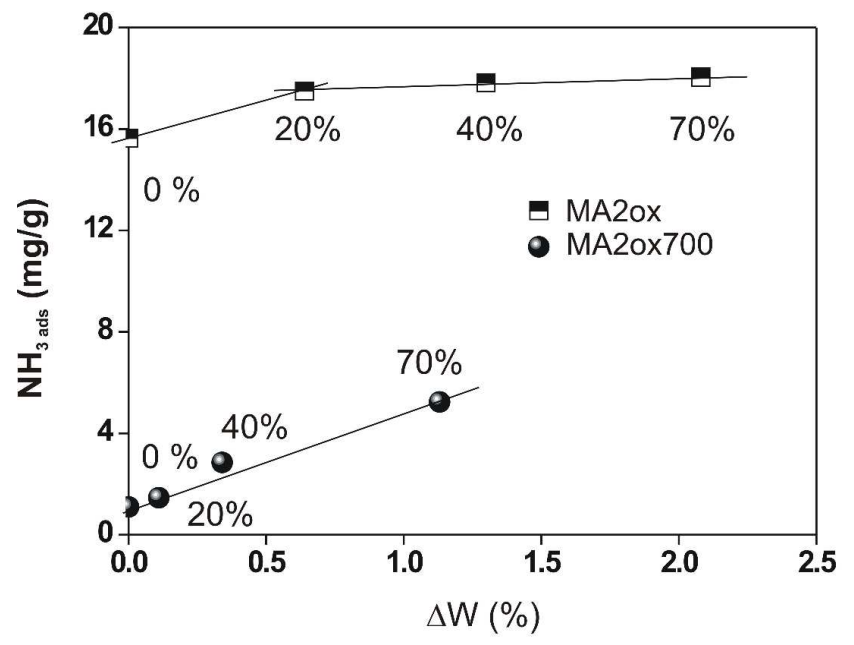<smiles>NO</smiles>

$\mathrm{H}_{2} \mathrm{O}$

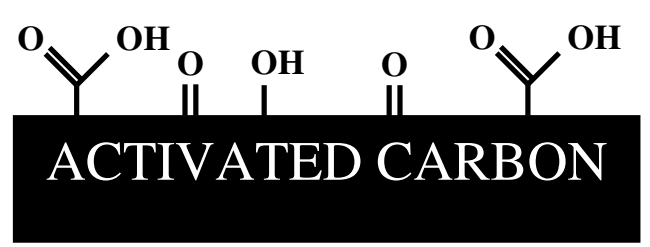

